\title{
Major health issues of American Indians
}

\begin{abstract}
This paper explores the topic of major health issues among a certain minority group. The inspiration of this paper comes from the authors personal family health history and past experiences with other members of his ethnic group and the same ethnic group this paper focuses on. This paper is concerned with the health of American Indians. Three different health issues that all have a different level or type of significance among the current state of health in American Indians. The overall effect of each health issue is explored and shown in a variety of ways throughout this document. This article addresses all the negatives of Diabetes Mellitus, Alcoholism/Substance Abuse, and Native American Myopathy in American Indians. Each separate health issue is broken down, explained in detail, and concentrates on the adverse effects each condition has on the race as a whole from physical, social, and emotional states. This paper presents the findings of studies and analysis from doctors and researchers across a variety of fields. The sources used to support the author's point were gathered using the internet. The articles and evidence used from the articles are peer-reviewed. The sources as a whole cover each specific topic of this paper.
\end{abstract}

Keywords: alcoholism, american indians, diabetes mellitus, native american myopathy, prevalence, substance abuse
Volume 5 Issue 3 - 2018

\author{
Jarett E Raade, Prabir K Mandal \\ Edward Waters College, USA
}

Correspondence: Prabir K Mandal, Edward Waters College, Biology Program, I 658 Kings Road, Jacksonville, FL 32209, USA, Tel (904)470-809I, Email Prabir.mandal0807@ewc.edu

Received: April 25, 20I8 | Published: June 28, 2018

\section{Introduction}

American Indians are the original inhabitants of the land what is now called the United States of America. There was once between 50 to 100 million American Indians in this land, a part of numerous different tribes spread from the great north east coast through the plains all the way to the rocky beaches of the west.

"American Indians and Alaska Natives born today have a life expectancy that is 4.4 years less than the U.S. all races-population (73.7 years to 78.1 years, respectively)". ${ }^{1}$

This paper explores the major health issues among the American Indian population of the United States of America. This paper covers three major health conditions and other negative factors brought on by each; Diabetes Mellitus, the genetic disorder Native American Myopathy, and Alcoholism and other drug addictions among American Indians. All of these health conditions not only are very prevalent among American Indians but have higher mortality rates in American Indians when compared to other races or the general United States population.

\section{Diabetes mellitus}

Diabetes Mellitus is a disease that inhibits the human body from correctly or successfully using the energy that should be received from the food and drinks a person consumes. Diabetes mellitus can occur in primarily two ways. The first occurs when the pancreas either only produces a miniscule amount or no insulin at all. Insulin is a hormone that in healthy individuals occurs naturally and is produced by the beta cells of the pancreas. Insulin is a hormone that enables the human body to turn sugar into energy. This type of diabetes is commonly referred to as Type 1 diabetes. Type 1 diabetes occurs because the beta cells that are produced by the pancreas are damaged and otherwise not working. Beta cells are what actually create insulin and without insulin (or very little), sugar is unable to be properly implemented into the human body's cells for use as energy. People with Type 1 diabetes have to use injections of artificial insulin in order to control their blood glucose or more commonly referred to as blood sugar. According to the Cleveland Clinic, Type 1 diabetes is most commonly associated with people living with diabetes who are under the age of 30 , but it can occur at any age and around ten percent of all diabetes diagnosis are Type $1 .^{2}$

Another way diabetes occurs is when a healthy amount of insulin is produced from the pancreas but, the insulin is not effective as it is either not enough or the insulin does not function properly. This is called insulin resistance and referred to more prominently as Type 2 diabetes. The Cleveland Clinic reports that $90 \%$ or Nine out of 10 people diagnosed with diabetes have Type 2 . Type 2 diabetes occurs more frequently in people who are both over the age of 40 and also are overweight or obese. ${ }^{2}$ Type 2 diabetes in a majority of situations can be controlled with the right combination of diet, weight management, and exercise. In more serious cases necessary treatment may include oral glucose-lowering medications or insulin injections in similar to that of Type 1 diabetes patients. The Indian Health Service, reports that Type 2 diabetes is the most common type of diabetes in American Indian and Alaska Native people and also in similar to the general population that the Cleveland Clinic refers to, this type of diabetes can occur at any age including, young children. ${ }^{3}$

Well in to the 1950's Diabetes Mellitus was virtually non-existent among American Indians. "As recently as 1955, diabetes was unrecognized as a leading cause of death as evidenced by its absence in a listing of the 10 most frequent causes of death for this population. However, by 2009, diabetes had jumped to number four on the list". ${ }^{4}$

As of 2010, American Indians and Alaska Natives now have the highest Diabetes Mellitus diagnosis rates of all racial and ethnic groups in the United States including non-Hispanic whites. According to the American Diabetes Association, over 16\% of all American Indians have been diagnosed and live with diabetes. This is in comparison to a rate of only $8.7 \%$ of non-Hispanic whites. The American Diabetes Association also reports that the Pima Indians of the U.S. State of Arizona have the highest rates of diabetes in the world, with more than $50 \%$ having a diagnosis of type 2 diabetes. ${ }^{4}$ 
According to data from the Indian Health Services, American Indians and Alaska Natives undergo long-term complications of diabetes at a higher prevalence and long-term complications develop earlier in life than that of any other ethnic group. Cardiovascular disease (CVD) is the number one cause of death in the United States among the general population. American Indians and Alaska Natives with diabetes are three to four times more likely to develop CVD than those without diabetes. Research also shows that diabetes is the leading cause of new blindness, end-stage renal disease, and lowerextremity amputation. ${ }^{4}$

According to the Indian Health Service, American Indians have a 63.6 age-adjusted mortality rate concerning deaths attributed to Diabetes Mellitus. ${ }^{1}$ This number is believed to include conditions and/or complications that are brought on from having the disease, Diabetes Mellitus. The overall age-adjusted mortality rate concerning diabetes mellitus deaths among the general United States population is 21.0. When comparing the two drug induced mortality rates American Indians have a higher rate at just over 3 deaths to every one death by the general United States of America population regardless of ethnic group.

Another alarming thing about the prevalence of diabetes in American Indians is the fact that diabetes is becoming more frequently prevalent among American Indian children and young adults. Data from the Indian Health Service, showed a 160\% increase in diagnosed diabetes for American Indians between the ages of 25 and 34 years between 1990 and 2004.

"In children under the age of 15 years of age, there was a $77 \%$ increase during this time period, and there were 128 and 94\% increases, respectively, for those who were 15-19 and 20-24 years of age". ${ }^{4}$

There are many different facets that are contributing factors to the alarming rates of diabetes in American Indians and Alaska Natives that include genetic, environmental, and behavioral issues, which may include a genetic predisposition toward insulin resistance, exposure of fetuses to hyperglycemia during pregnancy, sedentary lifestyles, obesity, and the effects of living in environments that are stressproducing, from both a social and physical standpoint.

American Indian culture is heavily based on customs and practices of each individual tribes' ancestors and the ways of living elders. Each individual tribe or band of American Indians has their own daily practices and beliefs. Since each tribe is individually unique perceptions and beliefs about medical issues including diabetes vary considerably from one tribe to another. It is crucial that diabetes care providers recognize this individualism because individuals' understanding and beliefs about the origins of diabetes will affect how, why, and if they seek treatment.

\section{Native American myopathy}

Native American Myopathy (NAM) is a genetic disease as well as a genetic disorder caused by a unique gene mutation that almost exclusively effects American Indians. Myopathy is the general term that oversees both a large and diverse group of diseases that can all be defined as a muscle disease. Native American Myopathy in like of other forms of myopathy is caused when certain muscle fibers are genetically mutated. ${ }^{5}$ The direct effect of Native American Myopathy is muscle fibers decline to correctly contract compared to a healthy individual. ${ }^{6}$ American Indians have one of the highest rates of any form of myopathy when measured up to other ethnic groups. Data suggests that individuals with blood lines in tribes located in the southern half of the United States of America are more susceptible to Native American Myopathy in comparison to Native American Myopathy rates in tribes in the northern half of the United States. An example of this is found in the Lumbee Tribe of Indians where 1 in 5000 have Native American Myopathy.

Humans carry two copies of every gene in their DNA sequence. One copy or allele of each gene or genetic trait is passed down to each of their offspring. Native American Myopathy is a recessive genetic disease. All recessive genes need to have both copies of the recessive gene in order to be present in an individual. This is unlike dominant genes where only one copy or allele would need to be passed down to offspring from either parent. There are also genes that are sex linked in the forms of X-linked dominant, $\mathrm{X}$-linked recessive and Y-linked. Females only carry $\mathrm{X}$ chromosomes and therefore have two copies of all X-linked genes. They can be homozygous or heterozygous for any of them. Males are XY and carry only one copy of X and one of Y. The fact that males only carry one $\mathrm{X}$ chromosome leaves them vulnerable to mutations on the $\mathrm{X}$ chromosome compared to females who carry two copies. ${ }^{7}$

The fact that a gene mutation is to blame for Native American Myopathy is a very recent discovery in terms of overall scientific discoveries. The gene mutation was not discovered in humans first but in Zebrafish. It was discovered that Zebrafish have mutation in their Stac3 gene. This gene was originally thought to be exclusively tided to muscle development and had no previous connection to regular muscle function in any species or muscle contraction in particular. Conversely, it was discovered by researchers that the Stac3 gene is responsible and a necessity for aiding in the transmission of signals from the species brain into muscle contractions. ${ }^{8}$ This process is called excitation-contraction coupling.

Native American Myopathy had been previously been placed to a select segment of the human genome but geneticist and other scientific research professionals had been previously unable to select which gene out of several hundred is liable for the genetic disorder. However, more extensive research has been conducted that determined that in similarity to the Zebrafish that the Stac3 gene was also responsible for excitation-contraction coupling in humans. Researchers have found five separate Lumbee Indian extended-families that reported a history of Native American Myopathy in their bloodlines. The families were recruited to see if any family members living with Native American Myopathy had mutations to the Stac3 gene. The results concluded that every individual with NAM carried the exact same mutation in both copies of their Stac3 genes, while their parents had a mutation in one copy of their Stac3 gene. This has led researchers to conclude that a mutation in Stac3 gene is the cause of Native American Myopathy. ${ }^{9}$

Here is a more in detail explanation of how the Stac3 gene relates to Native American Myopathy. "The loss of Stac3 resulted in a progressive breakdown of myofibers during larval stages with apparent swollen SR observed by 7 days' post fertilization. Given the myopathic features of mutants, we explored whether Stac 3 mutations might cause congenital human myopathies. Human STAC3 mapped to chromosome 12q13-14, and its specific location was within the previously defined genetic locus for the congenital NAM". ${ }^{10}$ This study used zebrafish larvae due to the common Stac3 gene mutation related to myopathy. 
Native American Myopathy can be depicted through many different symptoms that are variant in severities. However, there are certain characteristics that are more common than others such as Cleft palate, drooping facial features (resulting from relaxed facial muscles), and a short height in physically-mature adults. It should also be noted Individuals living with Native American Myopathy are also susceptible to Malignant Hyperthermia which poses a great concern. Malignant Hyperthermia is a life-threatening condition marked by constant overheating which often leads to heat stroke. Malignant Hyperthermia also exposes individuals to accelerated heart rate. The most severe problem is malignant hyperthermia often can be triggered by exposure to anesthetic drugs. This contributes to serious complications and often is lethal during a surgery if a physician is blind or otherwise unknowing to a patient having Malignant Hyperthermia brought on by their genetic mutation on the Stac 3 gene. ${ }^{9}$

Native American Myopathy may not be as common as other diseases but nonetheless has a very adverse effect on individuals but also their families as it is a very serious and sometimes fatal disease that is genetic in nature. Native American Myopathy research has had recent breakthroughs with the identification of which gene is mutated. The next step in research is trying to find a way to reverse the mutation completely.

\section{Alcoholism/drug addiction}

American Indians have a 23.9 age-adjusted mortality rate concerning drug-induced deaths. This does not include conditions that are brought on from drug usage but deaths directly related to the drugs themselves such as drug over-doses. The overall age-adjusted mortality rate concerning drug-induced deaths among the general United States population is 12.6. When comparing the two drug induced mortality rates American Indians have a higher rate at almost (1.9:1) 2 deaths to every one death by the general United States of America population regardless of ethnic group.

American Indians are approximately 1.7\% (5.2 million people) of the United States population and when taking this small percentage of people in to account they make up a large amount of substance abusers in the United States. It can be concluded that American Indians have the highest rates of substance abuse and related substance-use disorders. According to the National Survey on Drug Use and Health, nearly $9.2 \%$ of Native Americans ages 12 and older were abusers of alcohol. This is the highest rate (by a large margin) of any ethnic group including non-Hispanic whites. ${ }^{11}$ The Past-month underage binge drinking rate (people who noted binge drinking in the past month when survey was conducted) was $14.3 \%$ for American Indian and Alaska Native youth a higher rate than the $13.8 \%$ national average. The rate of illegal drug use in the last month among American Indians and Alaska Natives ages 12 and up in 2014 was $14.9 \%{ }^{12}$ To further illustrate the mortality rate given previously, in 2010, Native Americans had the highest rate of drug-induced death at $17.1 \% .^{12}$

"A comparison of two studies revealed that American Indian youth also exhibit a much higher prevalence of drug and alcohol use in 8th and 10th graders compared to national averages. According to the studies during the years 2009-2012, 56.2 percent of American Indian $8^{\text {th }}$ graders and 61.4 percent of those in 10th grade used marijuana in comparison to 16.4 percent of 8 th graders and 33.4 percent of 10th graders at a national average. ${ }^{13}$ The studies also revealed to be two to three times higher with the use of illicit substances, such as, heroin and oxycontin used by American Indian students compared to national averages in those years". ${ }^{14}$

According to the US Census, for the years 2007-2011 citizens of low economic status were primarily persons of American Indians, Black, or Hispanic/Latino heritage and totaled up to estimated totals of 42.7 million people. This is approximately $14.3 \%$ of the overall United States population. When further breaking down of $14.3 \%$, American Indians/Alaskan Natives were shown to have a poverty rate of $27 \%$. In the same survey those identifying as white had a poverty rate of $11.6 \%$. It can be concluded that with American Indians making up over a quarter of the population of United States living in poverty and over double that of whites living under poverty, all while only making up 1.7 of the overall population of the United States. It can be concluded that with so many American Indians living under impoverish conditions that the environment these individuals live in is lower in education levels but high in stress from not having money to pay for basic needs such as food and clothes making them more susceptible to substance abuse.

A significant factor to why American Indians struggle with addiction is they fail to properly complete (or complete in general) treatment programs. In accordance to the Substance Abuse and Mental Health Services Administration, less than 9 percent (8.9\%) of people aged 12 or older who needed treatment for alcohol abuse were able to receive specialized treatment to aid their problematic use of alcohol. ${ }^{11}$ The common source for incompletion of traditional treatment programs for substance abuse is due to cultural beliefs. "Traditionally these programs are almost exclusively based in Western theism and European-American cultural values and often African Americans, Hispanics/Latinos, and American Indians are unaffected by these programs due to their cultural differences. In some cases, these programs can also have a reverse effect on minorities leading them to undergo further trauma and/or harm". ${ }^{14}$

The chronic and excessive use of narcotics, alcohol and other illicit substances is known to lead to changes in mental state and even severe mental disorders. Substance abuse and its direct correlation on mental health of American Indians has been studied by researchers in the past. Mental disorders and other signs of poor mental health have been seen to have a significant prevalence among American Indians shown when compared to that of the national average. According to the data presented in the 2014 National Survey on Drug Use and Health (NSDUH), 21.2\% American Indians and Alaska Natives above the age of 18 shown at least one type of mental illness or severe lack of mental health in the past-year. ${ }^{15}$ American Indians and Alaska Natives above the age of 18 also showed a $4 \%$ rate of serious mental illness. ${ }^{12}$ When looking at the study in further detail it also revealed that $8.8 \%$ of American Indians and Alaska Natives ages 18 and up had both a mental disorder and a substance use disorder simultaneously. This is more than double the national average when compared to the national average of $3.3 \%$ in 2014 .

When looking at the leading causes of preventable death in the U.S. a whole population, alcoholism is the number three leading cause of preventable death. It is noted that $3.5 \%$ of all preventable deaths in the U.S. are ultimately determined to be alcohol-related. ${ }^{16}$ Besides, abuse of alcohol leading to mental health issues, abuse of alcohol can also lead to a large amount of problems within the liver and overall liver inflammation. Such conditions caused from a large amount of stress put on the liver include but are not limited to Alcoholic 
Hepatitis and Alcohol Cirrhosis. Alcohol Cirrhosis can eventually lead to liver failure, and then death. There is also data to show that Alcohol-related traffic deaths occur with a heightened frequency among Native Americans or Alaska Natives drivers in comparison to other than among other ethnic groups.

American Indians have been most affected by alcohol abuse, specifically, with slight inclusion of other substances. The alarming conclusion met, was that American Indian youth, use and abuse most drugs at a much higher rate and at a younger age, when compared to their peers, including the white ones.

\section{Conclusion}

Diabetes, Substance Abuse, and Native American Myopathy are all very devastating to a minority ethnic group that was once the only type of people on the land, but is seeing individual languages and whole tribes on the verge of going extinct largely in part to growing health issues like diabetes, native american myopathy, and alcoholism/ substance abuse.

The urgent need for diabetes prevention in American Indians can be summarized by a straightforward but concerning prediction from the Center of Disease Control. The CDC predicted that one in two American Indian/Alaska Native children born in 2000 will have type 2 diabetes in their lifetime unless the current trend is halted.

The development in Stac3-mutant zebrafish has the possibility to lead to possible experiments into determining treatments to native american myopathy through medication and therapies. The eventual goal is to create a drug that could reverse the defect/mutation to the muscular system in humans and particularly American Indians altogether.

American Indians have been most affected by alcohol abuse, specifically, with slight inclusion of other substances. The alarming conclusion met, was that American Indian youth, use and abuse most drugs at a much higher rate and at a younger age, when compared to their peers, including the white ones. This trend continues into adulthood and leads to major not only physical but also significant mental health issues.

\section{Acknowledgements}

We would like to give thanks to all the past research that has been conducted on each health issue presented. We would also like to acknowledge all of our family members and ancestors that have had to live through health issues as an American Indian.

\section{Conflict of interest}

The author declares that there is no conflict of interest.

\section{References}

1. Indian Health Service. Disparities. Fact Sheets; 2017.

2. What Is Diabetes Mellitus: Symptoms \& Treatment. Cleveland Clinic: Health Library; 2017.

3. Indian Health Service. Diabetes. Health Topics; 2017.

4. McLaughlin S. Traditions and Diabetes Prevention: A Healthy Path for Native Americans. Diabetes Spectrum. 2010;23(4):272-277.

5. Raade J. Native American Myopathy. 2017.

6. Scott Alan. Native American Myopathy. OMIM; 2013.

7. Cummings Michael R. Human Heredity. 11th ed. Australia: Cengage learning; 2016.

8. Huang SH, Hsiao CD, Lin DS, et al. Imaging of zebrafish in vivo with second-harmonic generation reveals shortened sarcomeres associated with myopathy induced by statin. PLoS One. 2011;6(9):e24764.

9. Akpan Nsikan. Genetic Clue Found For Native American Myopathy, Devastating Muscle Disorder. Medical Daily; 2013.

10. Horstick EJ, Linsley JW, Dowling JJ, et al. Stac3 is a component of the excitation-contraction coupling machinery and mutated in native american myopathy. Nat Commun. 2013;4:1952.

11. Alcohol. SAMHSA-Substance Abuse and Mental Health Services Administration. 2015.

12. Racial and Ethnic Minority Populations. SAMHSA - Substance Abuse and Mental Health Services Administration. 2018.

13. Substance Use in American Indian Youth is Worse than We Thought. USA: National institute on drug abuse; 2014.

14. Hickmon, M, Raade J. Addictions in Specific Minority Groups. 2017.

15. Minority Health and Health Disparities. National Institute on Alcohol Abuse and Alcoholism (NIAAA). 2017.

16. Mokdad AH, Marks JS, Stroup DF, et al. Actual causes of death in the United States, 2000. JAMA. 2004;291(10):1238-1245. 